\title{
Modeling Gate Leakage in InAs/AlSb HEMTs
}

\author{
M.G. Ancona, J.B. Boos, N. Papanicolaou, W. Chang, B.R. Bennett and D. Park \\ Electronics Science and Technology Division \\ Naval Research Laboratory \\ Washington, DC, USA \\ ancona@estd.nrl.navy.mil
}

\begin{abstract}
To simulate gate leakage in InAs/AISb HEMTs, we create a detailed model within the framework of density gradient theory that incorporates the entire InAs/AISb heterostructure, the quantum confinement and non-parabolicity effects in the InAs well and generation/recombination at the Type II InAs/AISb heterojunction (via both the spatially indirect band-to-band process and interface traps). Each of these ingredients is described individually and they are then put together as a model of gate leakage. Comparisons of preliminary leakage simulations with experiment suggest that interface traps are essential for understanding the behavior especially at low voltages.
\end{abstract}

Keywords-component; InAs/AlSb HEMTs; density gradient theory; gate leakage; Type II heterojunctions; non-parabolicity.

\section{INTRODUCTION}

Because of its wide range of bandgaps and band offsets and its high electron and hole mobilities, the InAs/AlSb/GaSb material system, nearly lattice-matched at $6.1 \AA$, is a very attractive candidate for future low-power, high-speed semiconductor technologies. Currently, both HEMTs and HBTs are being widely investigated and in the case of InAs/AlSb HEMTs excellent performance has been obtained including an intrinsic $\mathrm{f}_{\mathrm{T}}$ of $250 \mathrm{GHz}$ at $\mathrm{V}_{\mathrm{DS}}$ of $600 \mathrm{mV}$ and an $\mathrm{f}_{\mathrm{T}}$ of $90 \mathrm{GHz}$ at a $\mathrm{V}_{\mathrm{DS}}$ of only $0.1 \mathrm{~V}[1]$. One of the critical issues limiting the utility of state-of-the-art InAs/AlSb HEMTs is their relatively high gate leakage currents [2]. In order to investigate this issue we have carried out a combined experimental and simulation study in which we focus on the simplified HEMT gate diode depicted in Fig. 1 rather than on the sophisticated heterostructures actually used which have, for example, various extra barrier, dopant and sub-channel layers as well as source/drains. For the simple diode that we study the leakage current was measured to be as shown in Fig. 2.

An empirical understanding of the leakage in InAs/AlSb HEMTs has been given previously in [3]. Their understanding, arrived at using temperature-dependent leakage current measurements, was for a simpler structure than that in Fig. 1 having only a single layer of $\mathrm{AlSb}, \mathrm{AlGaSb}$ or $\mathrm{AlAsSb}$ interposed between the gate and channel. Under forward bias the I-V was a simple exponential and this was readily associated with hole injection over the fixed barrier at the Schottky contact. The reverse characteristic was more complicated with at least two kinks that they explained as due to (i) an overcoming of a valence band barrier induced by a Fermi level pinning at the Schottky contact and (ii) a rise in series resistance associated with channel depletion. This picture of the leakage characteristics is consistent with the data of [3] and seems reasonable es-

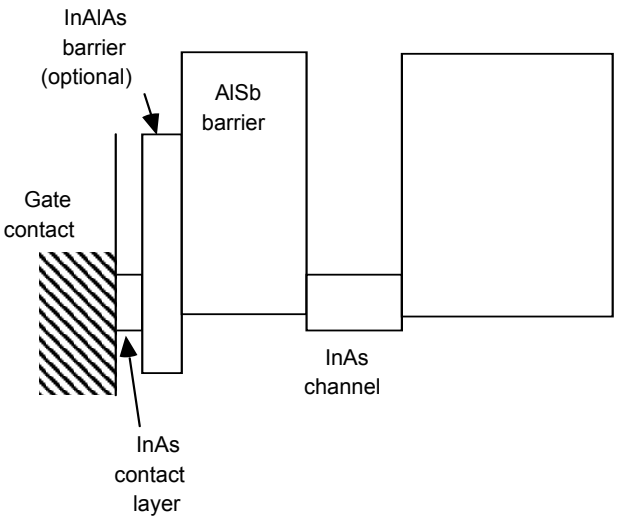

Figure 1. Band diagram of simplified HEMT heterostructure design.

pecially in the forward direction. However, it does not provide a quantitative understanding of, for example, the hole generation and it does not address what might happen in more practical device structures.

From a device modeling standpoint the problem of InAs/AlSb gate leakage is quite challenging because of the wide variety of physical phenomena that must be represented. The primary difficulty is that both classical and quantum transport physics are involved. In particular, as discussed in [3] and as seen by the effect of the InAlAs layer in Fig. 2, the leakage is dominated by classical hole transport over the relatively low

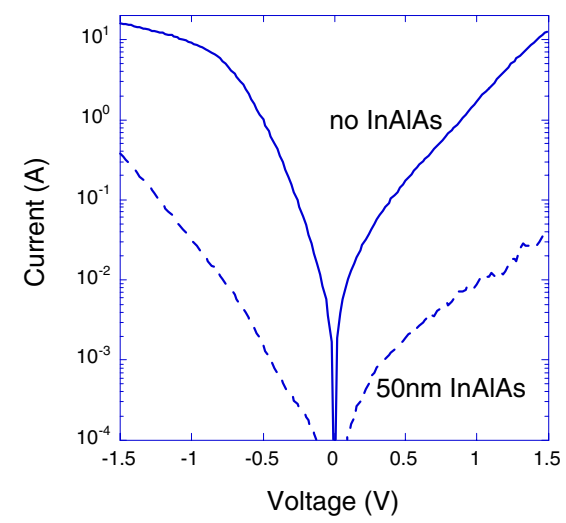

Figure 2. Experimental gate leakage characteristics for the diode like that in Fig. 1 with or without the InAlAs layer..

The authors thank DARPA and ONR for funding support. 
valence band barriers of the system. Yet at the same time quantum effects are also critical both in the carrier confinement in the InAs quantum well and in the spatially indirect generationrecombination processes at the Type II heterojunctions. In this paper we present a first effort to develop a detailed transport model using density gradient theory that incorporates most of the relevant device physics, both classical and quantum. The ultimate goal is to provide a more quantitative understanding of the gate leakage that might then suggest strategies for its reduction.

\section{THEORY}

\section{A. Density-Gradient Theory}

Density gradient (DG) theory is an approximate quantum transport theory that has come into wide use in semiconductor device simulation [4-6]. One of its main attractive features is that it allows both classical and quantum transport phenomena to be modeled simultaneously using a single, consistent set of equations that represent a physically well founded approximation to the quantum transport physics that are applicable in a variety of circumstances. Thus, DG theory would seem to be ideally suited for modeling InAs/AlSb gate leakage.

As physics, DG theory is a continuum or hydrodynamic description of the electron (or hole) gas. It is a generalization of diffusion-drift (DD) theory with the primary new ingredient being that the equation of state of the electron gas is made to depend not only on the gas density but also on the densitygradient. Having both dependences makes the electron gas response non-local (in a Taylor sense) and allows for a lowestorder representation of quantum mechanical non-locality. In simplest form the equation of state of DG theory (for the electron gas) is

$$
\varepsilon_{\mathrm{n}}=\varepsilon_{\mathrm{n}}^{0}(\mathrm{n})-\frac{\mathrm{b}_{\mathrm{n}}}{2} \frac{\nabla \mathrm{n} \cdot \nabla \mathrm{n}}{\mathrm{n}^{2}} \quad \text { where } \quad \mathrm{b}_{\mathrm{n}}=\frac{\hbar^{2}}{4 \mathrm{~m}_{\mathrm{n}}^{*} \mathrm{qr}_{\mathrm{n}}}
$$

where $\varepsilon_{\mathrm{n}}^{0}$ is the usual density-dependent part of the equation of state, $b_{n}$ is the DG coefficient and $r_{n}$ is a numerical constant whose value depends on the problem (see below). Based on (1), it can then be shown that the transport equation, a generalization of the diffusion-drift current equation, is

$\mathbf{J}_{\mathrm{n}}=-\mathrm{n} \mu_{\mathrm{n}} \mathbf{E}-\mathrm{D}_{\mathrm{n}} \nabla \mathrm{n}+2 \mathrm{~b}_{\mathrm{n}} \mu_{\mathrm{n}} \nabla\left(\frac{\nabla^{2} \mathrm{~s}}{\mathrm{~s}}\right) \quad$ where $\quad \mathrm{s} \equiv \sqrt{\mathrm{n}}$

This equation has been used to model a variety of device problems involving quantum confinement and quantum tunneling phenomena in semiconductors and even metals [4-6].

\section{B. Quantum Confinement}

The ability of DG theory to properly represent quantum confinement in heterostructures was explored in some detail in [7] for equilibrium situations. The results of that paper show that DG theory cannot be expected to provide an accurate approximation for the InAs quantum wells of a HEMT (e.g., Fig. 1) because of its narrow width $(100 \AA)$ and small mass $\left(\mathrm{m}_{\mathrm{e}}^{*} \sim\right.$ 0.023 ). Nevertheless, in [7] it was argued that one might still employ DG theory as an accurate phenomenology with $b_{n}$ parameterizing the effects of higher-order gradient terms. Be- cause of the preliminary nature of this paper we have, however, chosen to ignore this issue and instead simply use the theory with $r_{n}=1$.

Because of the high density of electrons and their small mass, non-parabolicity is quite important in the InAs HEMT channel as has been explored in detail in [8]. For the present work, we require an expression for the density-dependent part of the equation of state that can account for the effect of the non-parabolicity on the electron gas response. A simple form for the density-dependent part of the chemical potential (derivable from $\left.\varepsilon_{\mathrm{n}}^{0}(\mathrm{n})\right)$ that we have found reasonable is

$$
\varphi_{\mathrm{n}}^{\mathrm{o}}(\mathrm{n})=\mathrm{kT} \ln \left(\frac{\mathrm{n}}{\mathrm{N}_{\mathrm{C}}}\right)\left[\mathrm{a}_{1}+\mathrm{a}_{2}\left(\frac{\mathrm{n}}{\mathrm{N}_{\mathrm{C}}}\right)^{\mathrm{a} 3}\right]
$$

where $\mathrm{a}_{1}=1.04, \mathrm{a}_{2}=0.272$ and $\mathrm{a}_{3}=0.37$. This function is seen in Fig. 3 to compare quite well with the microscopic result obtained in [8]. It should be noted that the slight deviation of $a_{1}$ from unity was required for the best fit at high density, however, at low densities it means this expression does not satisfy the Maxwell-Boltzmann limit exactly.

Because the InAs and AlSb are not perfectly latticematched, strain in the InAs plays a role in determining the electronic structure in the channel [8] and its effect on the electron gas equation of state should therefore be considered. In this paper we have chosen to ignore this strain effect because of the unknown degree of strain relaxation in the actual device structure. Our simulations thus correspond to the situation of a fully relaxed InAs layer.

\section{Generation-Recombination}

As discussed in [3] and in the Introduction, to understand gate leakage in InAs/AlSb HEMTs is to understand the processes of hole generation and subsequent transport. With regards to hole generation there are at least three different mechanisms that must be considered:

Bulk generation/recombination. As discussed in [4], bulk generation-recombination in the InAs is treated differently in DG theory because $n_{\text {eq }} p_{\text {eq }}$ is no longer a known quantity as it is in DD theory but instead must be found by solving differential equations for equilibrium.

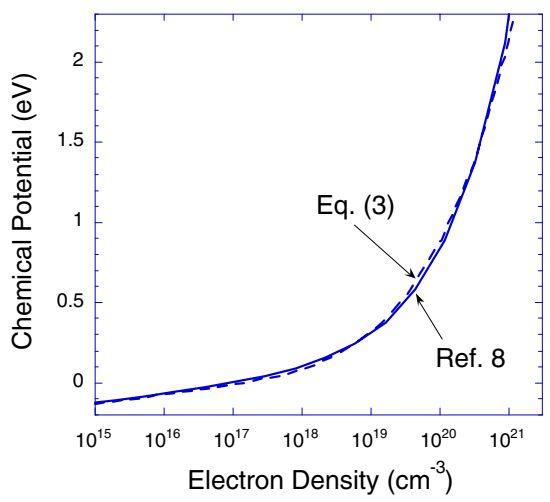

Figure 3. Chemical potential of electron gas in an InAs quantum well as computed in Ref. 8 compared with the curvefit of (3). 
Interface state generation/recombination. It is believed that interface traps are a significant presence at the InAs/AlSb interface and that the generation-recombination associated with them can play an important role in determining the characteristics of heterojunction bipolar transistors formed in this material [9]. A full treatment of the processes involved would involve a DG version of the conventional DD approach [10] and would require knowledge of the interface trap density and crosssections versus energy. Because these quantities are unknown, for this work we employ a simpler approach in which discontinuities in the electron and hole currents are introduced at the interface that are assumed proportional to the interface trap density.

Type II interface generation/recombination. It is known from photoluminescence measurements [11] that spatially indirect electron transitions occur at the Type II AlSb/InAs heterojunction. These transitions occur because of and in proportion to the degree of wave function overlap between electrons in the InAs conduction band and holes in the AlSb valence band. Such overlap requires barrier penetration by the carriers as shown schematically in Fig. 4 where two complementary processes for creating/annihilating electron-hole pairs at the Type II heterojunction are indicated. The requisite barrier penetrations are of course non-classical effects that cannot be analyzed within DD theory. They can be treated using DG theory, however, as we do here for the first time. Because DG theory provides a consistent treatment of the barrier penetration of the carriers we expect it to allow the critical effects of doping, bias and confinement on the generation to be analyzed and understood.

Finally, a fourth process, hole generation via impact ionization in the channel, is not considered here because we analyze only the HEMT gate diode and do not treat the situation of HEMT operation with source-drain current flow.

\section{Channel Resistance}

When the gate electrode is reverse biased the InAs channel becomes depleted and therefore its series resistance rises. When this series resistance becomes an appreciable fraction of the gate resistance, then a significant voltage drop will occur along the channel and the gate leakage problem will become two dimensional with different parts of the surface having, in effect, different gate biases. We perform only one-dimensional

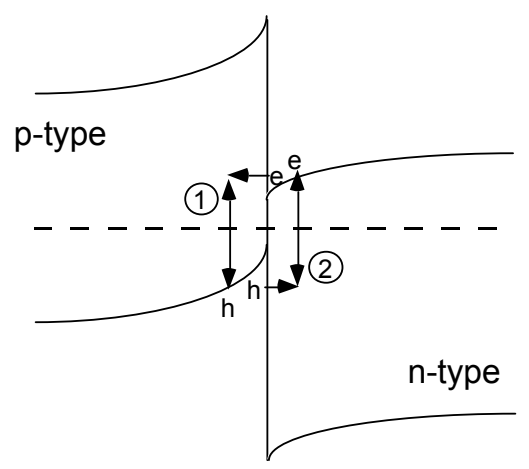

Figure 4. Schematic depicting two interband processes at a Type II heterojunction. simulations in this paper and so an accurate treatment of the channel resistance effect is impossible.

\section{RESULTS AND DISCUSSION}

As a first test problem we consider a p-AlSb/n-InAs heterodiode with the active layers being of the same thickness as in a typical HEMT and with contacting regions that block minority carriers. A plot of the computed band diagram and carrier density profiles in Fig. 5 shows that electrons from the InAs do indeed penetrate into the AlSb. The Figure also shows that the second process of tunneling of holes from the AlSb into the InAs is negligible compared to the holes that cross the $0.1 \mathrm{eV}$ valence band offset via a thermionic process. In Fig. 6 we plot the I-V characteristics of the pn diode under forward bias assuming no interface trap generation and that either the bulk or interface generation mechanism is active with the rates set by the radiative process [12]. For the set of parameters used here the bulk and interface generation mechanisms curiously produce currents of similar magnitude with interface generation

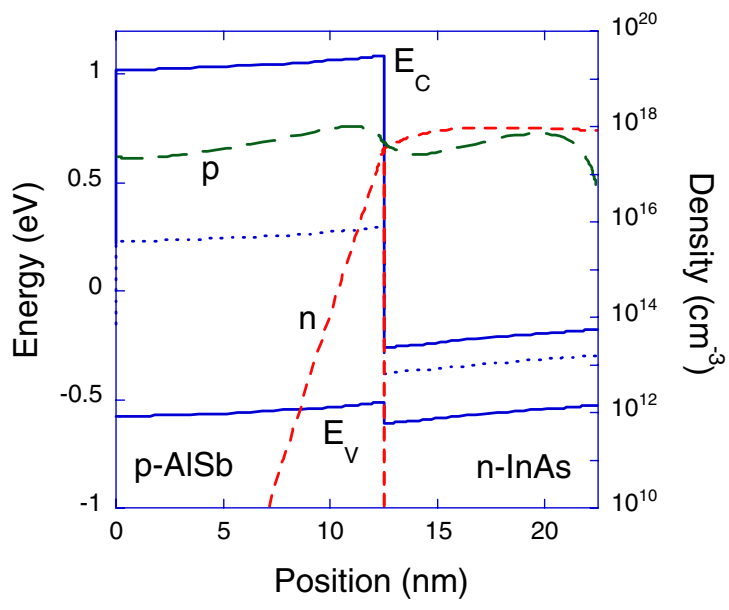

Figure 5. Band diagram and carrier density and recombination rate profiles in a $\mathrm{p}-\mathrm{AlSb} / \mathrm{n}-\mathrm{InAs}$ heterodiode with $\mathrm{V}=0.5 \mathrm{~V}$.

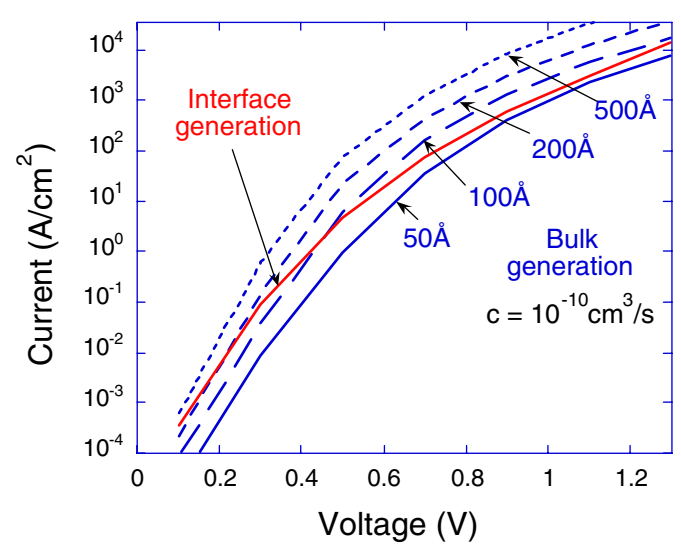

Figure 6. Computed generation currents in a $\mathrm{p}-\mathrm{AlSb} / \mathrm{n}-\mathrm{InAs}$ heterodiode as a function of bias. 
being larger at low voltages but smaller at higher voltages and with the magnitude of the bulk current depending on the channel thickness.

Next, employing the same parameters as in Figs. 5 and 6 , we apply DG theory to the structure of Fig. 1 without the InA1As layer, with an ohmic contact made to the channel carriers and an offset voltage at the Schottky contact of $180 \mathrm{mV}$ [3]. A computed band diagram is shown in Fig. 7 for an applied bias of $0.5 \mathrm{~V}$. This diagram shows the diode band structure and band bending as computed with DG theory. In Fig. 8 we plot the computed gate leakage characteristics both with and without interface traps at the InAs/AlSb interfaces along with the experimental data from Fig. 2. In the forward direction at high voltage, the I-V characteristic is roughly exponential as was found in [3]. However, at lower voltage, the simulation underestimates the current by a significant amount unless interface traps are included (with a density chosen to give a good fit). Under reverse bias the observed behavior is completely unexplainable without interface traps at all voltages. (The saturation of the simulated curves at high negative bias is simply due to the channel depletion not being treated properly in our 1-D simulations as was explained earlier). The effect of interface traps in these simulations is readily understood if one notes that the hole current is determined by both the rate of hole generation and by the barriers in the structure that can block these holes. At low voltage, the carrier distributions are nearequilibrium and therefore the generation rates are low and limit the current unless interface traps are present. At higher (positive) voltages, the generation rates rise and become high enough that even without traps the current is barrier-limited.

Despite the considerable uncertainties in the various material parameters, the results reported herein are intriguing and they suggest that DG theory can be used to analyze the main processes that are responsible for HEMT gate leakage.

\section{REFERENCES}

[1] J.B. Boos et al., "0.1mm AlSb/InAs HEMTs with InAs subchannel," Electron. Lett., vol. 34, pp. 1525-1526, 1998; J.B. Boos et al., "Ohmic contacts in AlSb/InAs high electron mobility transistors for low-voltage operation,” J. Vac. Sci. Technol. B, vol. 17, pp. 1022-1027, 1999.

[2] J.B. Boos et al., "AlSb/InAs HEMTs with a TiW/Au gate metalization for improved stability,” Solid-St. Electron., vol. 47, pp. 181-184, 2003.

[3] B. Brar and H. Kroemer, "Hole transport across the $(\mathrm{Al}, \mathrm{Ga})(\mathrm{As}, \mathrm{Sb})$ barrier in InAs-(Al,Ga)(As.Sb) heterostructures,” J. Appl. Phys., vol. 83, pp. 894-899, 1998.

[4] M.G. Ancona and H.F. Tiersten, "Macroscopic description of the silicon inversion layer," Phys. Rev., vol. B35, pp. 7959, 1987; M.G. Ancona, "Macroscopic description of quantum-mechanical tunneling," Phys. Rev., vol. B42, pp. 1222-1233, 1990; M.G. Ancona, Z. Yu, R.W. Dutton, P.J. Vande Voorde, M. Cao and D. Vook, "Density-gradient analysis of MOS tunneling,” IEEE Trans. Elect. Dev., vol. 47, pp. 2310$2319,2000$.

[5] A. Wettstein, A. Schenk and W. Fichtner, "Quantum device simulation with the density-gradient model on unstructured grids," IEEE Trans. Elect. Dev., vol. 48, pp. 279-284, 2001; D. Connelly, Z.P. Yu and D. Yergeau, "Macroscopic simulation of quantum mechanical effects in 2D MOS devices via the density gradient method," IEEE Trans. Elect. Dev., vol. 49, pp. 619-626, 2002.

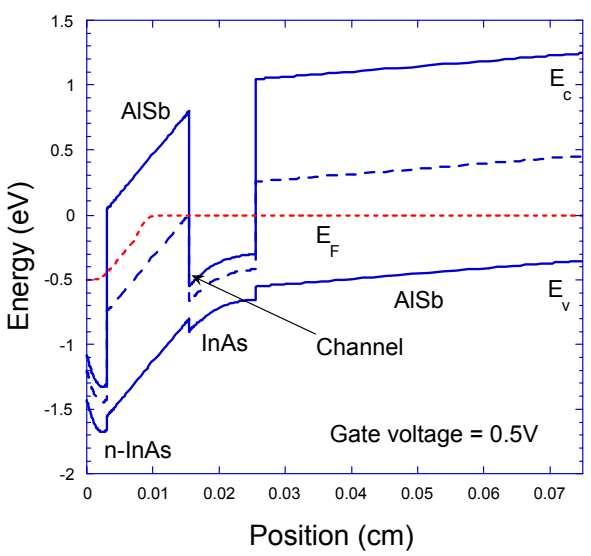

Figure 7. Band diagram of the HEMT diode computed by DG theory.

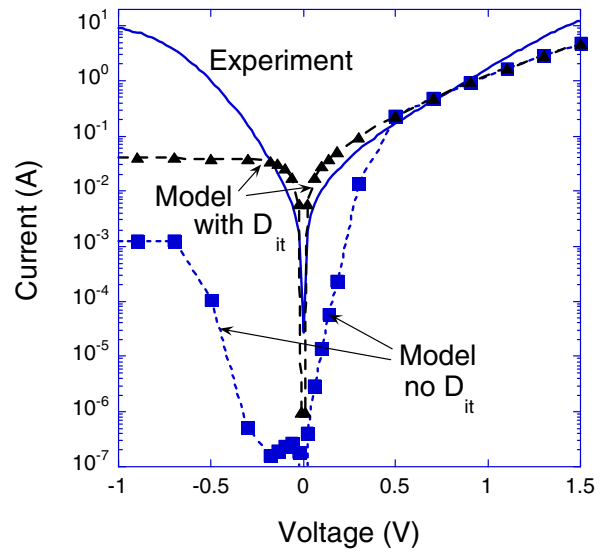

Figure 8. Comparison of experimental and simulated gate leakage characteristics with and without interface traps.

[6] The density gradient equations (in the scattering-dominated limit [4]) have been implemented in the commercial software tools from Integrated Systems Engineering, Inc. and from Silvaco, Inc.

[7] M.G. Ancona,'Density gradient theory analysis of electron distributions in heterostructures," Superlatt. Microstruct., vol. 7, pp. 119-130, 1990; "Finite temperature density-gradient theory," Proc. Workshop Comp. Electron., p. $113,1992$.

[8] P.J. Lin-Chung and M.J. Yang, "Subband structures of strained AlSb/InAs/AlSb quantum wells,” Phys. Rev., vol. 48, pp. 5338-5344, 1993.

[9] H. Kroemer, C. Nguyen and B. Brar, "Are there Tamm-state donors at the InAs-AlSb quantum well interface?," J. Vac. Sci. Technol. B., vol. 10, pp. 1769-1772, 1992; J.J. Pekarik, H. Kroemer and J.H. English, “An AlSb-InAs-AlSb double-heterostructure P-n-P bipolar transistor," J. Vac. Sci. Technol. B, vol. 10, pp. 1032-1034, 1992.

[10] J.G. Simmons and L.S. Wei, "Theory of dynamic charge current and capacitance characteristics in MIS systems containing distributed surface traps," Solid-State Electron., vol. 16, pp. 53-66, 1973.

[11] F. Fuchs, J. Schmitz, J.D. Ralston and P. Koidl, "Spatially direct and indirect photoluminescence from InAs/AlSb heterostructures," Phys. Rev., vol. B49, 13638-13642, 1994.

[12] M. Levinshtein, S. Rumyantsev and M. Shur, Semiconductor Parameters, Vol. I. Singapore: World, 1996, p. 159. 